\title{
Role of community-based resources in cervical cancer screening uptake in low- and middle-income countries: a scoping review protocol
}

\author{
Danladi Adamu ${ }^{1}$ (D) ${ }^{\text {a }}$, Nicole M Robertson ${ }^{2}$, David Weller ${ }^{3}$, Christine Campbell ${ }^{3}$ \\ 1 Usher Institute, Teviot Place, University of Edinburgh, EH8 9AG, UK; College of Medical Sciences, Gombe State University, Nigeria, ${ }^{2}$ Usher Institute, \\ Teviot Place, University of Edinburgh, EH8 9AG, UK; University of Kentucky College of Medicine, Lexington, Kentucky, USA, 3 Usher Institute, Teviot \\ Place, University of Edinburgh, EH8 9AG, UK \\ Keywords: low- and middle-income countries, community-based resources, protocol, Imics, screening, cervical cancer \\ https://doi.org/10.29392/001c.18246
}

\section{Journal of Global Health Reports}

Vol. 4, 2020

\begin{abstract}
Background
Poor cervical cancer screening coverage and utilization by women in low- and middle-income countries (LMICs) are linked to health system factors such as lack of access and availability of quality health care, inadequate workforce capacity, and socio-cultural and behavioral barriers. To improve women's participation in cervical screening, strategies such as involvement of community resources have been recommended. The aim of this review is to understand the current roles of community-based resources (CBRs) such as community health workers (CHWs) and community-based civil society organizations (CBOs) including key stakeholders - for example health champions, traditional leaders, chief's wives, etc. in the community in cervical screening in LMICs settings.
\end{abstract}

\section{Methods and analysis}

We will conduct a scoping review of MEDLINE, CINAHL and Global Health databases from January 2016 to June 2020 for published peer-reviewed literature from LMICs including reference list tracking and handsearching of studies with community focus published in English describing interventions provided by CBRs in cervical screening uptake.

\section{Ethics and dissemination}

Ethical approval is not required. Findings from this review will be summarised for conferences and published in peer-reviewed journals for widespread dissemination. Study will identify possible gaps in the evidence and differences in the role of CBRs between sub-Saharan Africa (SSA) and other LMIC regions and inform recommendations and implementation of future cervical screening research, policy, and practice.

Cervical cancer is a preventable and treatable disease when identified early. ${ }^{1}$ Cervical cancer in low- and middleincome countries (LMICs) accounts for about $85 \%$ of the global burden of cervical cancer. ${ }^{1}$ We define LMICs based on the 2020 World Bank classification of income economies. ${ }^{2}$ The high burden of cervical cancer in LMICs could be reduced through a comprehensive approach that involves prevention, early diagnosis, and effective screening and treatment programs. ${ }^{3}$ However, access to these programs in LMICs is limited by many challenges such as lack of policies and programs for cervical cancer, lack of resource allocation, lack of access and availability of quality cervical cancer prevention services, and inadequate manpower in LMICs. ${ }^{4}$
The World Health Organization (WHO) in response to these challenges published guidance in 2013, recommending that in settings where access to HPV vaccination and screening using cytology and colposcopy are not available, alternative evidence-based cervical screening methods such as HPV testing and visual inspection with acetic acid (VIA) by trained healthcare workers could be used to screen women. ${ }^{5}$

A number of LMICs such as Zambia, Bangladesh, Guatemala, Honduras, and Nicaragua have scaled up cervical screening to population level using these approaches. ${ }^{6}$ However, several challenges remain for expansion of screening, such as training and maintaining the cadre of healthcare personnel who can sustain screening and treat-

\footnotetext{
a Correspondence to: Danladi Adamu, Usher Institute, University of Edinburgh, Medical Quad, Teviot Place, Edinburgh, EH8 9AG, United Kingdom. Email: danladi.adamu@ed.ac.uk.
} 
ment, ensuring adequate follow-up for screen-positive women, and overcoming the lack of government commitment due to competing priorities with infectious and other non-communicable diseases. ${ }^{6}$ Women's lack of knowledge of cervical cancer and screening, and socio-cultural barriers may also affect acceptability and uptake of screening services. Therefore, further, alternative strategies for improving uptake and implementation of cervical cancer screening in LMICs are required. One additional strategy is the use of community-based resources (CBRs) as described previously in low-income settings, ${ }^{7-9}$ and involvement of nonbusiness and non-governmental civil society organizations including key individuals in the community. ${ }^{10,11}$ Involvement of the community can promote trust and improve acceptance of screening and cultural permissibility within the community. ${ }^{10-12}$

\section{RATIONALE}

Recently, systematic reviews by Driscoll ${ }^{13}$ and O'Donovan ${ }^{12}$ have shown that community health workers (CHWs) could help in reducing barriers and increase acceptance of cervical screening in LMICs. However, no review to date has been published examining the broader involvement of community-based resources including key stakeholders - for example health champions, traditional leaders, chief's wives, etc. in the community in cervical screening in LMICs settings.

\section{OBJECTIVES}

The review objectives are to assess: i) the role of community-based resources (CBRs) such as community health workers (CHWs) and community-based civil society organizations (CBOs) including key stakeholders - for example health champions, traditional leaders, chief's wives, etc. in the community in cervical screening in LMICs settings; and summarize the key findings where effectiveness of interventions utilizing CBRs is reported; and ii) to compare roles of CBRs across geographical regions.

\section{METHODS}

\section{SCOPING REVIEW FRAMEWORK}

We will adapt the methodological guideline articulated in Arksey and O'Malley's framework ${ }^{14}$ and further enhanced by Levac ${ }^{15}$ and Daudt ${ }^{16}$ for conducting and reporting this scoping review. The framework involves the following stages: i) identifying the research question ii) identifying relevant studies iii) study selection iv) charting the data, and v) collating, summarizing, and reporting the results.

\section{IDENTIFYING THE RESEARCH QUESTION}

The research questions are: 1) What are the roles of CBRs in cervical cancer screening in LMICs? and 2) Are there differences in the roles of CBRs in cervical screening across geographical regions?

\section{IDENTIFYING RELEVANT STUDIES}

We will search for published literature in English language between January 2016 and June 2020 for current and up to date information in the following 3 electronic databases: MEDLINE, CINAHL and Global Health based on their ability to capture the bulk of relevant LMICs literature. We will use keywords for exploring the above databases: cervical cancer, screening, community health workers, communitybased organisations, civil society organizations, HIV, and low- and middle-income countries. Medical Subject Heading $[\mathrm{MeSH}]$ and free text terms will also be developed and combined to identify published studies. Truncation commands (using root words to capture alternative word endings), proximity operators (for words within a chosen distance of each other) and Boolean logic operators (OR and AND) will be used, and to ensure highest yield, pilot trial with search terms will be carried out and refined. More papers will be located through handsearching of citations and reference list tracking and contacts with authors for further information. A broad range of search terms based on descriptions from previous papers by Adamu ${ }^{7}$ and O' Donovan ${ }^{17}$ to capture all relevant literature.

We searched International Prospective Register of Systematic Reviews (PROSPERO), Cochrane Library, PubMed, and Google scholar and no published or scheduled review on our topic was identified. Search strategy for MEDLINE via OVID is shown in Table 1: the search strategy will be adapted for other databases.

\section{STUDY SELECTION}

Relevant titles and abstracts identified from databases search will be uploaded and saved in EndNote X9 Library. After screening for duplicates, the remaining titles and abstracts will be independently screened by two reviewers based on the review's eligibility criteria (see Table 2) developed according to the research questions. Thereafter, fulltext copies will be downloaded, and the 2 reviewers will further screen the studies for eligibility of inclusion into the review and disagreements will be resolved by discussion, and $10 \%$ of the selected studies will be checked by $3^{\text {rd }}$ reviewer for consistency. Reasons for exclusion of studies screened in full text will be documented.

PRISMA flow chart diagram ${ }^{18}$ will be used to summarize the study selection.

\section{ELIGIBILITY CRITERIA}

PICOS (Population, Intervention, Comparison, Outcome and Study design) framework will guide the selection of eligibility criteria.

\section{CHARTING THE DATA}

Results from included studies will be extracted using a data extraction form (Online Supplementary Document, Table S1). The form will be piloted and updated and include the following items: study author, date, and study design; study population and country; name of CBRs; role of CBRs and screening modality; and key outcomes and comments. 
Table 1. MEDLINE search strategy via OVID MEDLINER (R)

\begin{tabular}{|c|c|c|}
\hline 1 & $\begin{array}{l}\text { (cervical screen } \$ \text { or cervical cancer or cervical neoplas } \$ \text { or cervical malignan } \$ \text { or colposcopy or HPV DNA or } \\
\text { Pap smear or Papanicolou or direct visualisation or liquid cytology or visual inspection).mp. [mp=title, } \\
\text { abstract, original title, name of substance word, subject heading word, floating sub-heading word, keyword } \\
\text { heading word, organism supplementary concept word, protocol supplementary concept word, rare disease } \\
\text { supplementary concept word, unique identifier, synonyms] }\end{array}$ & 96136 \\
\hline 2 & $\begin{array}{l}\text { organizations/ or "academies and institutes"/ or charities/ or faith-based organizations/ or labor unions/ or } \\
\text { organizations, nonprofit/ or public-private sector partnerships/ or self-help groups/ or societies/ }\end{array}$ & 65452 \\
\hline 3 & Civil society organisations.mp. & 86 \\
\hline 4 & 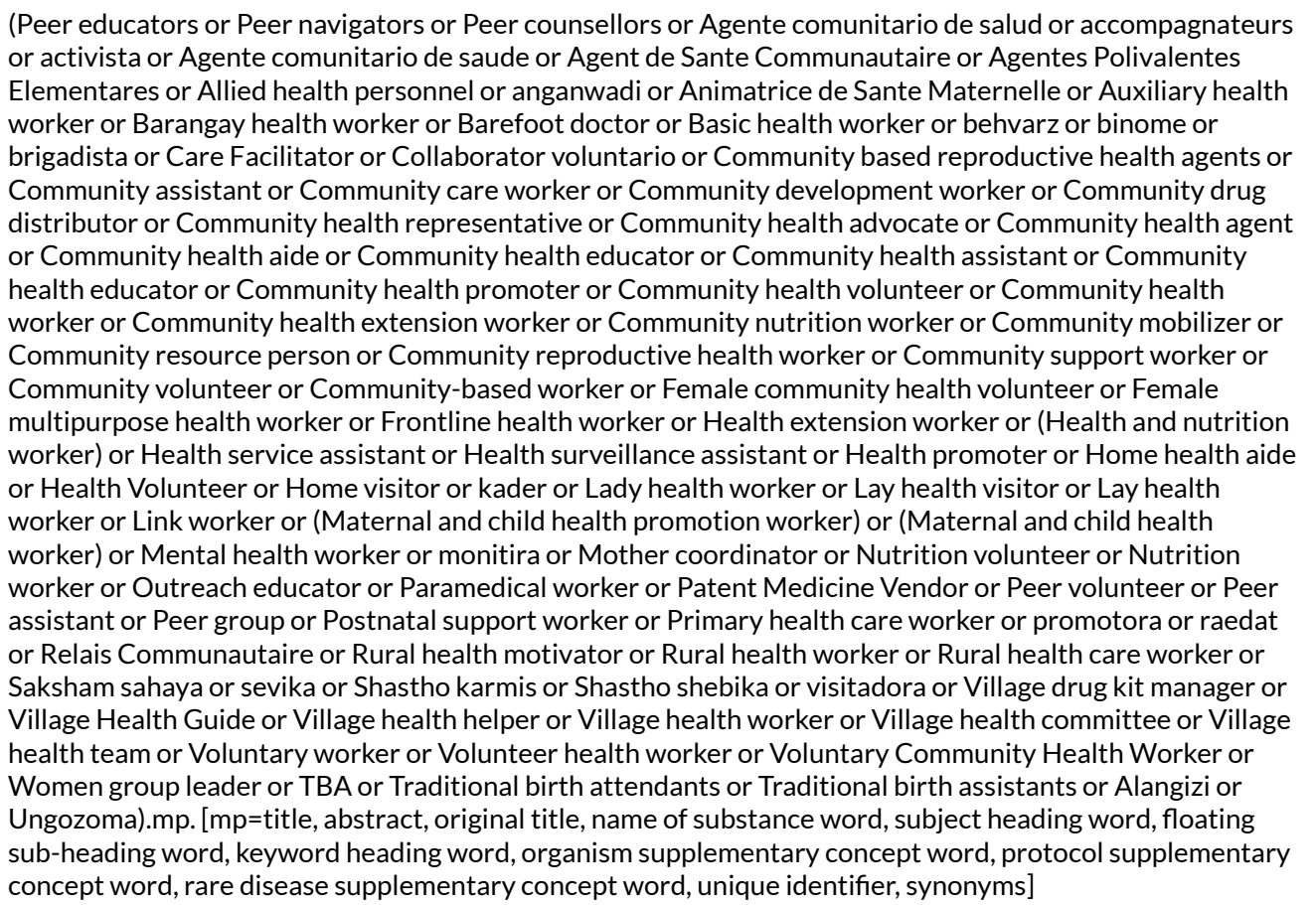 & 40448 \\
\hline 5 & 1 or 2 or 3 or 4 & 200914 \\
\hline 6 & $\begin{array}{l}\text { (Afghanistan or Albania or Algeria or American Samoa or Angola or Argentina or Armenia or Azerbaijan or } \\
\text { Bangladesh or Belarus or Belize or Benin or Bhutan or Bolivia or (Bosnia and Herzegovina) or Botswana or } \\
\text { Brazil or Bulgaria or Burkina Faso or Cape Verde or Cambodia or Cameroon or Chad or China or Central } \\
\text { African Republic or Colombia or Comoros or Congo or Congo democratic Republic or Costa Rica or Cote d } \\
\text { Ivoire or Cuba or Djibouti or Dominica or Dominican Republic or Ecuador or Egypt or El Salvador or } \\
\text { Equatorial Guinea or Eritrea or Eswatini or Ethiopia or Fiji or Gabon or Gambia or Georgia or Ghana or } \\
\text { Grenada or Guatemala or Guinea or Guinea-Bissau or Guyana or Honduras or India or Indonesia or Iran or } \\
\text { Iraq or Haiti or Jamaica or Kazakhstan or Kenya or Kiribati or Korea democratic republic or Kosovo or } \\
\text { Kyrgyz or Lebanon or Lao or Lesotho or Liberia or Libya or Madagascar or Malawi or Malaysia or Maldives or } \\
\text { Mali or Moldova or Marshall Islands or Mauritius or Mauritania or Micronesia or Mexico or Montenegro or } \\
\text { Mongolia or Morocco or Mozambique or Myanmar or Namibia or Nauru or North Macedonia or Nicaragua } \\
\text { or Niger or Nigeria or Pakistan or Papua New Guinea or Paraguay or Peru or Philippines or Romania or } \\
\text { Russia or Rwanda or Samoa or (Sao Tome and Principe) or Senegal Serbia or Sierra Leone or Somalia or } \\
\text { Solomon Islands or South Africa or South Sudan or Sri Lanka or Sudan or Suriname or Syria or Tajikistan or } \\
\text { Tanzania or Thailand or Timor-Leste or Togo or Tonga or Tunisia or Turkey or Turkmenistan or Tuvalu or } \\
\text { Uganda or Ukraine or Uzbekistan or Vanuatu or Venezuela or Vietnam or (West Bank and Gaza) or Yemen or } \\
\text { Zambia or Zimbabwe or Africa or Asia or Caribbean or West Indies or South America or Latin America or } \\
\text { Central America or developing or less developed or underdeveloped or middle income or low* income or } \\
\text { underserved or deprived or poor* or countr* or nation* or population* or world).mp. [mp=title, abstract, } \\
\text { original title, name of substance word, subject heading word, floating sub-heading word, keyword heading } \\
\text { word, organism supplementary concept word, protocol supplementary concept word, rare disease } \\
\text { supplementary concept word, unique identifier, synonyms] }\end{array}$ & 4337372 \\
\hline 7 & 5 and 6 & 56393 \\
\hline 8 & Limit 7 to English language & 49323 \\
\hline 9 & Limit 8 to yr="2016 - 2020" & 10105 \\
\hline 10 & Limit 9 to humans & 9797 \\
\hline
\end{tabular}


Table 2. Eligibility criteria

\begin{tabular}{|c|c|c|}
\hline $\begin{array}{l}\text { PICOS } \\
\text { element }\end{array}$ & Included & Excluded \\
\hline Population & $\begin{array}{l}\text { Are studies whose participants are: } \\
\text { - Mainly CBRs involved in supporting community-based cervical screen- } \\
\text { ing services in LMIC setting, } \\
\text { - Women aged } 18 \text { years and above eligible for screening in LMIC setting, } \\
\text { - Women either HIV positive or HIV negative, } \\
\text { - Women with prior normal screening results or scheduled for follow-up } \\
\text { - Women due or overdue for screening in settings such as screening pro- } \\
\text { grams, HIV clinics, community health centers, and reproductive health } \\
\text { centers. }\end{array}$ & $\begin{array}{l}\text { Are studies whose participants are: } \\
\text { - CBRs supporting community-based } \\
\text { cervical screening services outside } \\
\text { - LMIC setting, } \\
\text { - Women who have had hysterectomy. } \\
\text { - Women who were treated or are in fol- } \\
\text { - low-up care for cervical cancer. } \\
\text { - Wregnant women. } \\
\text { - Women younger than } 18 \text { years. }\end{array}$ \\
\hline Intervention & $\begin{array}{l}\text { - Services offered by CBRs with or without health care workers in cervi- } \\
\text { cal screening in LMIC setting. } \\
\text { - Services offered by CBRs with focus on both cervical screening and } \\
\text { other aspects of cervical cancer e.g. HPV vaccination. We will only ex- } \\
\text { tract and report findings for cervical screening. } \\
\text { - Services offered by CBRs within and outside of LMICs. We will only ex- } \\
\text { tract and report findings for LMICs. }\end{array}$ & $\begin{array}{l}\text { - Services offered by CBRs in cervical } \\
\text { screening outside LMIC setting. } \\
\text { - Services offered by health care work- } \\
\text { ers without CBRs in cervical screening. } \\
\text { - Services offered by CBRs on other as- } \\
\text { pects of cervical cancer, not related to } \\
\text { cervical screening e.g. HPV vaccina- } \\
\text { tion. }\end{array}$ \\
\hline Comparison & $\begin{array}{l}\text { Studies with or without control group, and the control groups are } \\
\text { women who either receive no intervention, another intervention other } \\
\text { than that of intervention group, or routine standard screening. }\end{array}$ & - \\
\hline Outcome & $\begin{array}{l}\text { - Role(s) played by CBRs in cervical screening uptake among women } \\
\text { with and without HIV. } \\
\text { - Evidence of uptake or non-uptake of cervical screening. }\end{array}$ & - \\
\hline $\begin{array}{l}\text { Study } \\
\text { design }\end{array}$ & $\begin{array}{l}\text { - Quantitative studies, } \\
\text { - Qualitative studies, } \\
\text { - Mixed-methods studies, } \\
\text { - Systematic reviews. }\end{array}$ & $\begin{array}{l}\text { - Conference abstracts without full-text } \\
\text { - } \text { study. } \\
\text { - Commentaries } \\
\text { - Study protocols } \\
\text { - } \text { Petters } \\
\text { Policy briefs }\end{array}$ \\
\hline
\end{tabular}

\section{COLLATING, SUMMARIZING, AND REPORTING OF THE} RESULTS.

The extracted data will be summarized and presented in line with the broader aims of the scoping review. We recognize that there will be reasonable variation in the roles, and outcome measures used in the included studies, but only limited synthesis (description and comparison).

The extracted data will be reported via tables of summary of roles of CBRs based on LMICs regions according to World Bank groupings ${ }^{19}$ (see Online Supplementary Document, Table S2 and Table S3 for CHWs and CBOs, respectively) including comments on any intervention effects.

Learning from previous scoping and rapid reviews, a scoping review unlike conventional systematic reviews place less emphasis on the methodological quality appraisal of included studies. ${ }^{12,20-22}$ Therefore, we will not subject included studies to quality assessment.

\section{DISCUSSION}

This will be the first review to explore the broader and unique contribution of the diverse communities in LMICs in cervical cancer screening. The review will summarise the current evidence on the roles of CBRs in cervical screening in LMICs from the international literature; comparison of these roles across geographical areas will be also be described.

Our search will be limited to published studies from January 2016 to June 2020 to map the landscape of current evidence without the constraints of rigorous analysis and synthesis of review findings; as such, we will restrict our search to 3 databases namely: MEDLINE, Global Health and CINAHL without considering the literature from non-peer reviewed and grey databases. Nevertheless, we are aware that limiting our search to peer reviewed English language publications within the last 5 years in 3 databases risks missing some papers.

Findings from this review will identify potential gaps in 
evidence and any differences in the roles of CBRs between countries in SSA and other LMICs.

\section{FUNDING}

DA was funded by Commonwealth Scholarship Commission. NR was supported by the Rotary International Global Fellowship from the Rotary Foundation. The Commission and Rotary Foundation had no role in preparation, funding, and decision to publish this paper.

\section{AUTHORSHIP CONTRIBUTIONS}

DA, DW and CC conceived this scoping review protocol.

DA drafted the manuscript, which was assessed and refined by NR, CC and DW.

NR is the second reviewer.

All authors approved the final version of the manuscript.

DA is the guarantor.

\section{COMPETING INTERESTS}

All authors have completed the Unified Competing Interest form available at http://www.icmje.org/conflicts-of-interest/ in line with the Journal of Global Health editorial policy and declare no conflicts of interest.

\section{CORRESPONDENCE TO:}

Danladi Adamu

Usher Institute, University of Edinburgh

Medical Quad, Teviot Place, Edinburgh, EH89AG, United Kingdom.

Email: danladi.adamu@ed.ac.uk

Submitted: September 29, 2020 GMT, Accepted: November 27, 2020 GMT

This is an open-access article distributed under the terms of the Creative Commons Attribution 4.0 International License (CCBY-4.0). View this license's legal deed at http://creativecommons.org/licenses/by/4.0 and legal code at http://creativecommons.org/licenses/by/4.0/legalcode for more information. 


\section{REFERENCES}

1. Bray F, Ferlay J, Soerjomataram I, Siegel RL, Torre LA, Jemal A. Global cancer statistics 2018: GLOBOCAN estimates of incidence and mortality worldwide for 36 cancers in 185 countries. $C A: A$ Cancer Journal for Clinicians. 2018;68(6):394-424. do $\mathrm{i}: 10.3322 /$ caac. 21492

2. TheWorldBank. World Bank Classication of income economies. https://datahelpdesk.worldbank.org/know ledgebase/articles/906519-world-bank-country-and-1 ending-groups. Accessed May 2, 2020.

3. World Health Organization. Comprehensive Cervical Cancer Control: A Guide to Essential Practice. Reproductive Health, World Health Organization. Chronic Diseases, \& Health Promotion; 2006.

4. World Health Organization. Improving Data for Decision-Making: A Toolkit for Cervical Cancer Prevention and Control Programmes.; 2018.

5. World Health Organisation. Guidelines for Screening and Treatment of Precacancerous Lesions for Cervical Cancer Prevention. http://www.who.int/e ntity/reproductivehealth/publications/cancers/screen ing_and treatment_of_precancerous_lesions/en/inde x.html. Accessed March 4, 2018.

6. Holme F, Kapambwe S, Nessa A, Basu P, Murillo R, Jeronimo J. Scaling up proven innovative cervical cancer screening strategies: Challenges and opportunities in implementation at the population level in low- and lower-middle-income countries. International Journal of Gynecology \& Obstetrics. 2017;138:63-68. doi:10.1002/iigo.12185

7. Adamu DB, Robertson N, Weller D, Campbell C. Implementation and Effectiveness of communitybased resources to increase cervical cancer screening uptake among women living in sub-Saharan Africa: A protocol for systematic review. Journal of Global Health Reports. 2019;3. doi:10.29392/joghr.3.e201905 $\underline{0}$

8. Wright J, Walley J. Future trends in global public health. Public health: An action guide to improving health. 2010:317-332.

9. Musesengwa R, Chimbari MJ. Community engagement practices in Southern Africa: Review and thematic synthesis of studies done in Botswana, Zimbabwe and South Africa. Acta Tropica. 2017;175:20-30. doi:10.1016/j.actatropica.2016.03.02 1
10. Agurto I, Arrossi S, White S, et al. Involving the community in cervical cancer prevention programs. International Journal of Gynecology \& Obstetrics. 2005;89(Suppl 2):S38-S45. doi:10.1016/i.ijgo.2005.0 $\underline{1.015}$

11. Brawley OW, Cowal SG. Civil society's role in efforts to control women's cancers. The Lancet. 2017;389(10071):775-776. doi:10.1016/s0140-6736(1 6) $31799-8$

12. O’Donovan J, O'Donovan C, Nagraj S. The role of community health workers in cervical cancer screening in low-income and middle-income countries: a systematic scoping review of the literature. BMJ Global Health. 2019;4(3):e001452. do i:10.1136/bmigh-2019-001452

13. Driscoll SD, Tappen RM, Newman D, VoegeHarvey K. Accuracy of visual inspection performed by community health workers in cervical cancer screening. International Journal of Gynecology \& Obstetrics. 2018;142(3):260-269. doi:10.1002/ijgo.125 $\underline{35}$

14. Arksey H, O’Malley L. Scoping studies: towards a methodological framework. International Journal of Social Research Methodology. 2005;8(1):19-32. doi:1 $\underline{0.1080 / 1364557032000119616}$

15. Levac D, Colquhoun H, O’Brien KK. Scoping studies: advancing the methodology. Implementation science. 2010;5(1). doi:10.1186/1748-5908-5-69

16. Daudt HM, van Mossel C, Scott SJ. Enhancing the scoping study methodology: a large, interprofessional team's experience with Arksey and O'Malley's framework. BMC medical research methodology. 2013;13(1):48. doi:10.1186/1471-228 8-13-48

17. O’Donovan J, O’Donovan C, Kuhn I, Sachs SE, Winters N. Ongoing training of community health workers in low-income and middle-income countries: a systematic scoping review of the literature. BMJ open. 2018;8(4):e021467. doi:10.1136/bmjopen-201 $\underline{7-021467}$

18. Moher D, Liberati A, Tetzlaff J, Altman DG, The PRISMA Group. Preferred reporting items for systematic reviews and meta-analyses: the PRISMA statement. PLoS Med. 2009;6(7):e1000097. doi:10.137 1/journal.pmed.1000097 
19. World Health Organisation. Definition of regional groupings. WHO Health statistics and information systems. https://www.who.int/healthinfo/global_burd en disease/definition regions/en/. Accessed May 26, 2020.

20. Githaiga JN, Walter FM, Scott SE, Mwaka AD, Moodley J. Symptom awareness measures for breast and cervical cancer in sub-Saharan Africa: A scoping review. SA Journal of Oncology. 2019;3(1):1-9.
21. Shrestha AD, Vedsted P, Kallestrup P, Neupane D. Prevalence and incidence of oral cancer in low - and middle - income countries: A scoping review. European Journal of Cancer Care. 2020;29(2):e13207. doi:10.1111/ecc. 13207

22. Haby MM, Chapman E, Clark R, Barreto J, Reveiz L, Lavis JN. What are the best methodologies for rapid reviews of the research evidence for evidenceinformed decision making in health policy and practice: a rapid review. Health research policy and systems. 2016;14(1):83. doi:10.1186/s12961-016-015 5-7 


\section{SUPPLEMENTARY MATERIALS}

\section{Online Supplementary Document}

Download: https://www.joghr.org/article/18246-role-of-community-based-resources-in-cervical-cancer-screeninguptake-in-low-and-middle-income-countries-a-scoping-review-protocol/attachment/47880.docx 